Draft Version OCtOBer 30, 2018

Preprint typeset using $\mathrm{IATE}_{\mathrm{E}} \mathrm{X}$ style emulateapj v. 12/16/11

\title{
GEMINI, SOFIA, AND ATCA REVEAL VERY YOUNG, MASSIVE PROTOSTARS IN THE COLLAPSING MOLECULAR CLOUD BYF 73
}

\author{
Rebecca L. Pitts ${ }^{1}$, Peter J. Barnes ${ }^{1,2}$, Stuart D. Ryder ${ }^{3,4}$, And Dan Li ${ }^{5}$ \\ Draft version October 30, 2018
}

\begin{abstract}
We present multi-wavelength data on the globally infalling molecular cloud/protostellar cluster BYF 73. These include new far-infrared (FIR) spectral line and continuum data from the Stratospheric Observatory for Infrared Astronomy's (SOFIA's) Far-Infrared Field-Imaging Line Spectrometer (FIFILS), mid-infrared (MIR) observations with the Thermal-Region Camera Spectrograph (T-ReCS) camera on Gemini-South, and 3mm continuum data from the Australia Telescope Compact Array (ATCA), plus archival data from Spitzer/Infrared Array Camera (IRAC), and Herschel/Photodetecting Array Camera and Spectrometer (PACS) and Spectral and Photometric Imaging Receiver (SPIRE). The FIFI-LS spectroscopy in [O I $] \lambda 63 \mu \mathrm{m},[\mathrm{O}$ III $] \lambda 88 \mu \mathrm{m},[\mathrm{O} \mathrm{I}] \lambda 145 \mu \mathrm{m}$, and [C II] $\lambda 158 \mu \mathrm{m}$ highlights different gas environments in and between the dense molecular cloud and Hir region. The photo-dissociation region (PDR) between the cloud and HII region is best traced by [O I] $\lambda 145 \mu \mathrm{m}$ and may have density $>10^{10} \mathrm{~m}^{-3}$, but the observed $\lambda 145 \mu \mathrm{m} / \lambda 63 \mu \mathrm{m}$ and $\lambda 63 \mu \mathrm{m} / \lambda 158 \mu \mathrm{m}$ line ratios in the densest gas are well outside model values. The HII region is well-traced by [C II], with the $\lambda 158 \mu \mathrm{m} / \lambda 145 \mu \mathrm{m}$ line ratio, indicating a density of $10^{8.5} \mathrm{~m}^{-3}$ and a relatively weak ionizing radiation field, $1.5 \lesssim \log \left(G / G_{0}\right) \lesssim 2$. The T-ReCS data reveal eight protostellar objects in the cloud, of which six appear deeply embedded $\left(A_{V}>30^{m}\right.$ or more) near the cloud's center. MIR 2 has the most massive core at $\sim 240 M_{\odot}$, more massive than all the others combined by up to tenfold, with no obvious gas outflow, negligible cooling line emission, and $\sim 3-8 \%$ of its $4.7 \times 10^{3} L_{\odot}$ luminosity originating from the release of gravitational potential energy. MIR 2's dynamical age may be as little as 7000 years. This fact, and the cloud's total embedded stellar mass being far less than its gas mass, confirm BYF 73's relatively early stage of evolution.

Keywords: infrared: ISM — submillimeter: ISM — stars: formation — stars: protostars — ISM: lines and bands
\end{abstract}

\section{INTRODUCTION}

The formation of massive star clusters is a topic of active debate and study (Longmore et al. 2014, and references therein). Current questions include the timescale and mechanisms of gas mass assembly and star formation (Peretto et al. 2013; Barnes et al. 2018), the degree to which gravity, turbulence, or magnetic fields control the dynamics (Crutcher 2012; Zamora-Avilés \& VázquezSemadeni 2014; Padoan et al. 2016; Kobayashi et al. 2018) and the fidelity with which we can measure these effects with only trace constituents (Pitts et al. 2018).

BYF 73 (= G286.21+0.17, part of the Galactic Census of High and Medium-mass Protostars (CHaMP) survey of molecular clouds; Barnes et al. 2010, 2011) is one of only a dozen or so known parsec-scale molecular clumps that are undergoing large-scale collapse/contraction, but where only a few protostars have formed so far, and the cloud is still gas-dominated (Peretto et al. 2013; Rygl et al. 2013; Wyrowski et al. 2016). With an estimated

\footnotetext{
rlpitts@ufl.edu

${ }^{1}$ Astronomy Department, University of Florida, P.O. Box 112055, Gainesville, FL 32611, USA

${ }^{2}$ School of Science and Technology, University of New England, Armidale NSW 2351, Australia

3 Department of Physics and Astronomy, Macquarie University, NSW 2109, Australia

4 Australian Astronomical Observatory, 105 Delhi Road, North Ryde, NSW 2113, Australia

${ }^{5}$ National Optical Astronomy Observatory, 950 North Cherry Avenue, Tucson, AZ 85719, USA
}

mass of $2 \times 10^{4} M_{\odot}$ and luminosity of $10^{4} L_{\odot}$, BYF 73 has the highest measured mass inflow rate, $0.034 M_{\odot} \mathrm{yr}^{-1}$, even among this extreme cohort (Barnes et al. 2010, 2016). Therefore, it may be in the early stages of forming a super star cluster like NGC 3603 in $\lesssim 0.5 \mathrm{Myr}$.

Such gas-dominated clouds are highly significant because the physical conditions, dynamics, and evolution must still be close to the cloud's initial state, as opposed to even slightly more evolved objects like hot cores (Gerner et al. 2014), where the internal conditions are already dramatically altered by the energy input from luminous protostars. Therefore, a careful study of each such cloud will provide important boundary conditions for star formation theory. In this Letter, we present a range of new and archival data on BYF 73 from $\mu \mathrm{m}$ to $\mathrm{mm}$ wavelengths, in order to examine the embedded protostellar content, gas mass distribution, and excitation conditions.

\section{OBSERVATIONS AND DATA REDUCTION}

\subsection{Spitzer/IRAC}

BYF 73 was observed by the Spitzer Space Telescope in all four IRAC bands $(3.6-8 \mu \mathrm{m})$ as part of the Galactic Legacy Infrared Midplane Extraordinaire (GLIMPSE) survey (Benjamin et al. 2003). We downloaded calibrated public data from NASA's IRSA website, and transformed to Galactic coordinates for ease of comparison with data at other wavelengths. We cropped these data to cover all emission associated with the molecular cloud and its adjacent compact His region, 


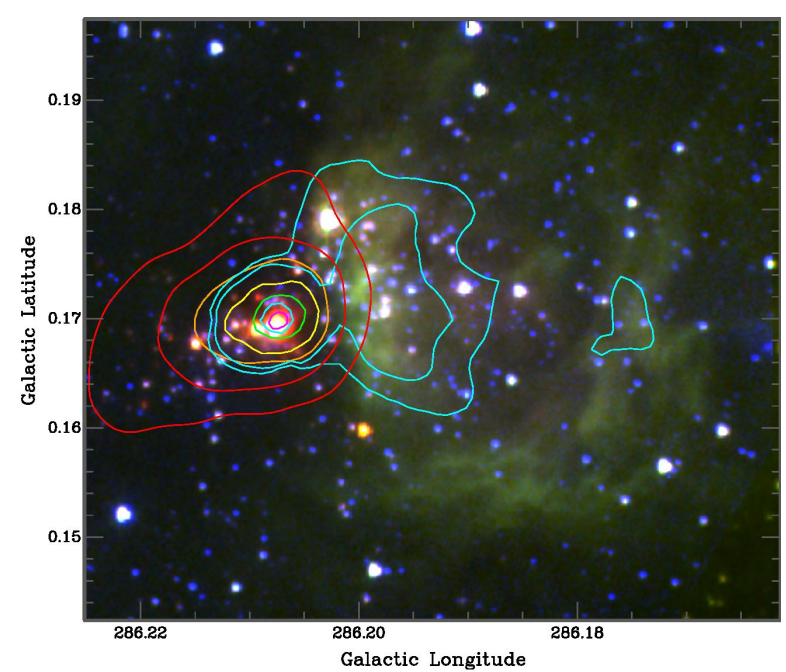

Figure 1. Composite image of Spitzer IRAC Bands 2 (red) and 1 (green), with $K$ (blue) from the Anglo-Australian Telescope's IRIS2 camera (Barnes et al. 2013). Contours are overlaid from Herschel and Australia Telescope Compact Array (ATCA) data: $500 \mu \mathrm{m}$ (red, at $30 \%$ and $50 \%$ of the peak value), $350 \mu \mathrm{m}$ (orange, $50 \%$ ), $250 \mu \mathrm{m}$ (yellow, 50\%), $160 \mu \mathrm{m}$ (green, 50\%), $70 \mu \mathrm{m}$ (cyan, $2 \%, 3 \%, 50 \%$ ), and $3 \mathrm{~mm}$ (magenta, $67 \%$ ). Note the clear separation between the molecular cloud to the left, and the compact HII region to the center and right.

as in the $\sim 4^{\prime}$ field shown in Fig. 1 . We measured the background-subtracted flux densities for the eight IRAC stellar sources that were also detected with the ThermalRegion Camera Spectrograph (T-ReCS; $\$ 2.2$ ); these are plotted in Fig. 3.

\subsection{Gemini-South/T-ReCS}

We obtained deep mid-infrared (MIR) data on BYF 73 with the high-resolution (point-spread function $(\mathrm{PSF}) \approx$ 0.3) T-ReCS camera (Telesco et al. 1998) at GeminiSouth on UT 2010 June $5-6$, as part of program GS2010A-Q-42 (PI: P. J. Barnes). The observations were set up to image a $1^{\prime}$ field of view with a six-field mosaic of the $20^{\prime \prime} \times 30^{\prime \prime}$ T-ReCS detector area, oriented to capture as many of the sources visible in the Spitzer images as possible. We cycled through the four filters $\mathrm{Si} 2$ (effective wavelength $8.74 \mu \mathrm{m})$, Si4 $(10.4 \mu \mathrm{m})$, Si6 $(12.3 \mu \mathrm{m})$, and Qa $(18.3 \mu \mathrm{m})$ during the observing to form a commensurate set of images and enable multi-band photometry of all sources. Cohen et al. (1999) MIR standard stars SAO 250905 and SAO 222647 were observed before and after BYF 73, respectively, for flux calibration. The data were reduced with an in-house MIR data reduction package, which performed the chop-and-nod correction and co-added frames to form one image of BYF 73 at each MIR wavelength. Reduced and flux-calibrated images are presented in Fig. 2 as a composite image of three of the four T-ReCS bands. The flux densities of the eight detected sources (based on aperture photometry with radii equal to three times the FWHM of the PSF) are included in the spectral energy distribution (SED) plot of Fig. 3. For MIR 1, these are totals for both components of an equal-brightness binary, separated by $0^{\prime \prime} .38$ $\left(\mathrm{PA}=-14^{\circ}\right)$ in the Si2 image, or $950 \mathrm{au}$ assuming a distance of $2.5 \mathrm{kpc}$ (Barnes et al. 2010). The other MIR sources show no evidence of binarity at this resolution.

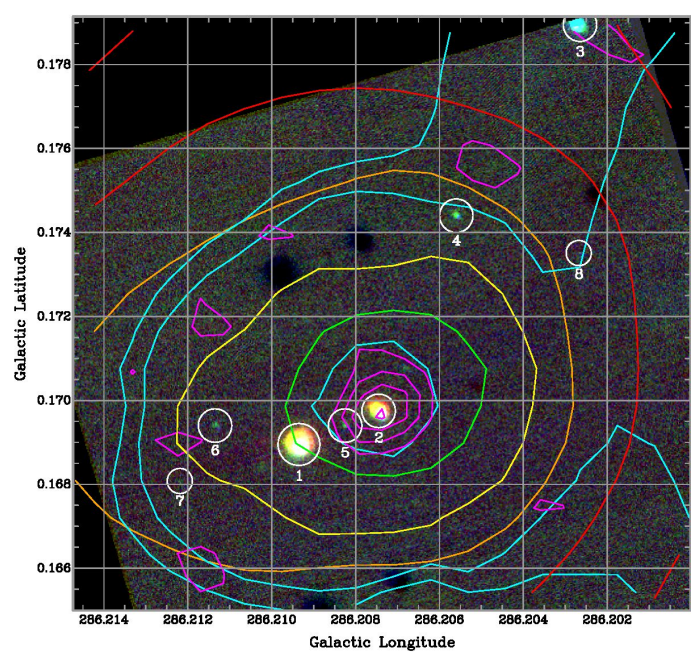

Figure 2. Identifications of point sources 1-8 detected in part of the $\sim 1^{\prime}$ wide Gemini-South/T-ReCS mosaic. This composite image has colors $(\mathrm{Qa}=$ red, $\mathrm{Si} 6=$ green, $\mathrm{Si} 2=$ blue $)$ indicating true flux ratio differences between these bands, and is overlaid by ATCA $3 \mathrm{~mm}$ continuum contours (magenta, from $14 \mathrm{mJy} / \mathrm{bm}$ spaced by $6 \mathrm{mJy} / \mathrm{bm}=1 \sigma)$ and the same far-infrared (FIR) contours as in Fig. 1. The "dark" sources are negative images of the bright ones, due to the $15^{\prime \prime}$ chop-throw between the target and reference positions in the telescope observing mode. Sources MIR 1, 2, and 5-7 were imaged in all three positions, and so appear as bright objects, flanked $15^{\prime \prime}$ to the N and S by dark images. Sources MIR 4, 8, and 3 (barely) were imaged in the target and one reference position.

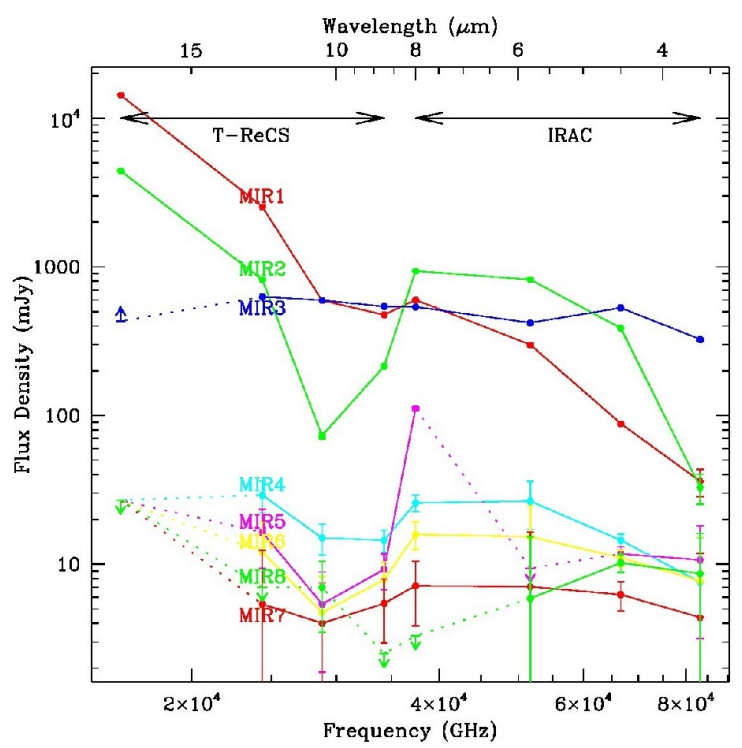

Figure 3. Flux densities of T-ReCS-detected sources in both TReCS and Spitzer/IRAC data. Secure detections are indicated by points connected by solid lines; $3 \sigma$ upper limits are indicated by symbols connected to the other data by dotted lines. As we obtained only a partial image for MIR 3 at $18.3 \mu \mathrm{m}$, its flux density is at least twice the indicated lower limit. Error bars are $1 \sigma$ noise values, but the calibration uncertainty is $\pm 15 \%$ for these data after allowing for subtraction of variable background emission.

\subsection{Stratospheric Observatory for Infrared Astronomy/Far-Infrared Field-Imaging Line Spectrometer (SOFIA/FIFI-LS)}

We obtained integral-field spectra of BYF 73 centered on the $[\mathrm{O} \mathrm{I}] \lambda 63 \mu \mathrm{m},[\mathrm{O} \mathrm{III}] \lambda 88 \mu \mathrm{m},[\mathrm{O} \mathrm{I}] \lambda 145 \mu \mathrm{m}$, and [C II] $\lambda 158 \mu \mathrm{m}$ lines on 2016 July $1 \& 3$ UT with FIFI-LS (Colditz et al. 2012; Klein et al. 2014) on board SOFIA, as part of project 04-0061 (PI: P. J. Barnes). Chop- 

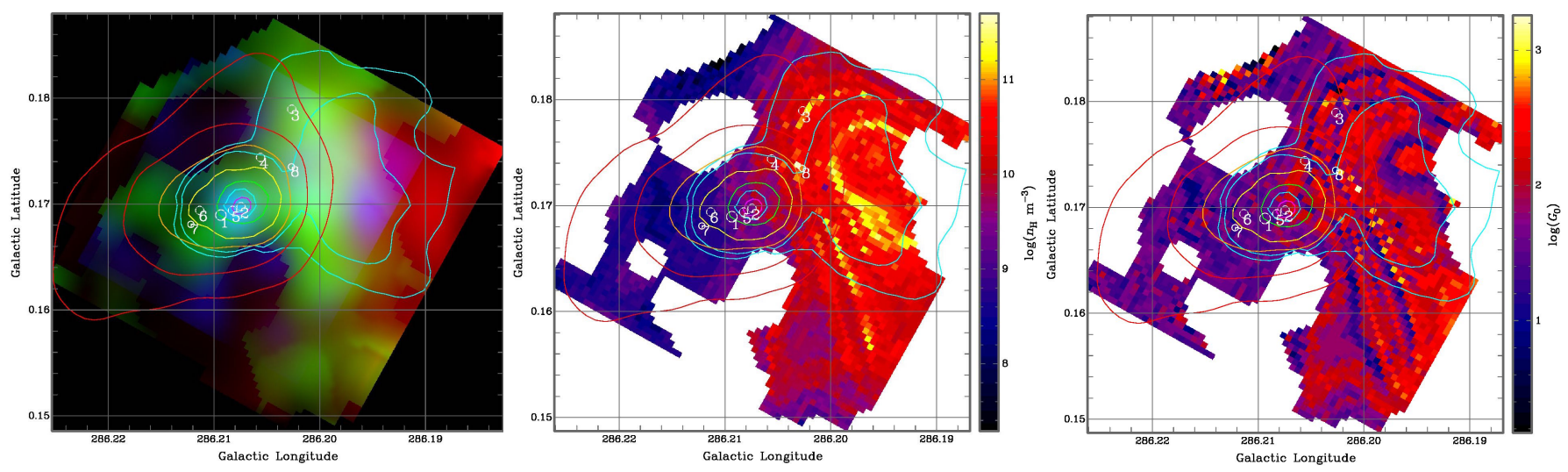

Figure 4. (Left) Composite image of $[\mathrm{C} \mathrm{II}] \lambda 158 \mu \mathrm{m}$ intensity (red, normalized to $3.8 \times 10^{-7} \mathrm{~W} \mathrm{~m}^{-2} \mathrm{sr}^{-1}$ ), $[\mathrm{O} \mathrm{I}] \lambda 145 \mu \mathrm{m}$ (green, $\left.6.4 \times 10^{-8} \mathrm{~W} \mathrm{~m}^{-2} \mathrm{sr}^{-1}\right)$, and [O I] $\lambda 63 \mu \mathrm{m}$ (blue, $\left.9.4 \times 10^{-11} \mathrm{~W} \mathrm{~m}^{-2} \mathrm{sr}^{-1}\right)$. (Middle and right) Respective images of derived log $\left(n_{\mathrm{H}}\right)\left(\mathrm{m}^{-3}\right)$ and $\log \left(G / G_{0}\right)\left(G_{0}\right.$ in Habing 1968 units, $\left.1.6 \times 10^{-6} \mathrm{~W} \mathrm{~m}^{-2}\right)$, computed by localizing FIFI-LS line intensities/ratios on the PDR Toolbox parameters. Contours in all panels are the same as in Fig. 1.

ping and nodding were done asymmetrically due to the many nearby MIR sources to the (Galactic) west and south. Each integral field spectrum combines over 200 exposures dithered in sub-pixel increments to boost the sampling of the final spatially resampled image cube. ${ }^{6}$ The total integration times were $1659 \mathrm{~s}$ centered on the $[\mathrm{C}$ II $] \lambda 158 \mu \mathrm{m}$ line, $3287 \mathrm{~s}$ on the [O I] $\lambda 145 \mu \mathrm{m}$ line, $1628 \mathrm{~s}$ on the $[\mathrm{O}$ III $] \lambda 88 \mu \mathrm{m}$ line, and $3318 \mathrm{~s}$ on the $[\mathrm{O} \mathrm{I}] \lambda 63 \mu \mathrm{m}$ line. Pipeline processing with FLUXER ${ }^{7}$ includes fitting and separation of line and continuum emission components in each band, and telluric correction.

\subsection{Herschel Photodetector Array Camera and Spectrometer (PACS) and Spectral and Photometric Imaging REceiver (SPIRE)}

We obtained archival Level 3.5 data from the Herschel (Pilbratt et al. 2010) satellite's PACS (Poglitsch et al. 2010) and SPIRE (Griffin et al. 2010) photometers, in order to combine with the other data described herein and fit SEDs across BYF 73 (see §3.2). The Herschel data were originally acquired as part of the Carina Nebula Complex (CNC) open time project (see Preibisch et al. 2012, for details).

\subsection{Atacama Submillimeter Telescope Experiment (ASTE) and $A T C A$}

The flux density at $850 \mu \mathrm{m}, 8 \pm 1 \mathrm{Jy}$, is from a singlepointing measurement at the line emission peak, made with the $10 \mathrm{~m}$ ASTE (Ezawa et al. 2004) as part of another project (Y. Yonekura 2016, private communication).

We observed BYF 73 at ATCA on 2010 October 1 and $4 \mathrm{UT}$ in the H75 array (baselines $\approx 31-89 \mathrm{~m}$ ) in both the $94 \pm 2 \mathrm{GHz}$ continuum and the $\mathrm{HCO}^{+}$and $\mathrm{H}^{13} \mathrm{CO}^{+}$ $J=1 \rightarrow 0$ emission lines $(89.19 \mathrm{GHz}$ and $86.75 \mathrm{GHz}$, respectively) as part of program C2288 (PI: Barnes). We used the quasars 0537-441 and 1045-62, respectively, as passband and complex gain calibrators, and Mars as the

\footnotetext{
${ }^{6}$ See the FIFI-LS GI handbook at https://www.sofia.usra.edu/ sites/default/files/FIFI-LS_GI_Handbook_RevB1.pdf, and the Cycle 5 SOFIA observer's handbook at https://www.sofia.usra.edu/ science/proposing-and-observing/sofia-observers-handbook-cycle$5 / 5$-instruments-ii-fifi-ls for details of the spectral \& spatial resolutions and observing modes.

${ }^{7}$ http://www.ciserlohe.de/fluxer/fluxer.html
}

flux calibrator. We mapped an 80-pointing mosaic of size 3 '.2 centered on the peak molecular line emission as measured in the Mopra maps (Barnes et al. 2010). Mediocre weather and poor phase stability, however, challenged the normal MIRIAD data reduction pipeline (Sault et al. 1995), resulting in line maps with low signal-to-noise ratios $(\mathrm{S} / \mathrm{N})$. The low spectral-line sensitivity was exacerbated by an apparently smooth intrinsic emission structure, as the ATCA $\mathrm{HCO}^{+}$line flux was $<30 \%$ of the Mopra single-dish value (Barnes et al. 2010), despite the short baselines. We fared better in the continuum, clearly detecting the point source MIR 2 at flux density $34 \pm 7 \mathrm{mJy}$ in the $55^{\prime \prime} 6 \times 44^{\prime \prime} 7$ synthesized beam (Fig. 2). MIR 3 may also have been detected, but at $\sim 2 \sigma$ this detection is not reliable; MIR 1 was not detected at all. Deconvolving MIR 2's measured size, we obtain $4{ }^{\prime \prime} 2 \times 3 . \prime \prime=10,600 \times 7400$ au at $2.5 \mathrm{kpc}$ for its physical dimensions at $3 \mathrm{~mm}$, consistent with MIR 2 being a massive protostellar core.

\section{ANALYSIS AND DISCUSSION}

\subsection{FIR Spectral Lines and Gas Conditions}

To analyze the FIFI-LS data, we localized the overlap of contours of two or more line ratios/intensities on $\log \left(n_{\mathrm{H}}\right)-\log \left(G / G_{0}\right)$ parameter maps from the Photo Dissociation Region Toolbox ${ }^{8}$ (PDRT; Kaufman et al. 2006; Pound \& Wolfire 2008). Fig. 4 shows the observed line intensity and derived $\log \left(n_{\mathrm{H}}\right)$ and $\log \left(G / G_{0}\right)$ maps, based on the PDRT and observed [C II] $\lambda 158 \mu \mathrm{m}$ and $[\mathrm{O} \mathrm{I}] \lambda 145 \mu \mathrm{m}$ line ratios. No line components were detected in $[\mathrm{O}$ III $] \lambda 88 \mu \mathrm{m}$. The $[\mathrm{O} \mathrm{I}] \lambda 63 \mu \mathrm{m}$ fluxes were difficult to reconcile with the fluxes in the [O I] $\lambda 145 \mu \mathrm{m}$ and $\left[\mathrm{C}_{\mathrm{II}}\right] \lambda 158 \mu \mathrm{m}$ lines, likely exacerbated by the [O I] $\lambda 63 \mu \mathrm{m}$ line's poor separation from the continuum in both the pipeline-processed spectra and our own alternative attempts. The $[\mathrm{O} \mathrm{I}] \lambda 145 \mu \mathrm{m} /[\mathrm{C} \mathrm{II}] \lambda 158 \mu \mathrm{m}$ ratio and integrated $[\mathrm{C} \mathrm{II}] \lambda 158 \mu \mathrm{m}$ line flux were enough to determine $n$ and $G / G_{0}$ along the PDR to about half a dex precision, as shown in Fig. 4 . There, we derive $\log \left(G / G_{0}\right) \approx$ $1.5-2$ and $n_{\mathrm{H}}$ ranging over $10^{10-11} \mathrm{~m}^{-3}$.

Outside of the PDR, especially near MIR 1-2, PDRT's built-in assumption of $A_{V} \leq 10^{m}$ breaks down, and there are no prescriptions for higher extinctions. $G / G_{0}$ did

\footnotetext{
${ }^{8}$ http://dustem.astro.umd.edu/pdrt/models1.html
} 
not noticeably change near MIR $1-3$, but $n$ fell where it was expected to rise. We found that the combination of weak [C II] $\lambda 158 \mu \mathrm{m}$ line emission (at levels PDRT that flags as unreliable) and moderately high $[\mathrm{O}$ I $] \lambda 145 \mu \mathrm{m} /[\mathrm{C}$ II $] \lambda 158 \mu \mathrm{m}$ ratios around MIR $1-3$ create contours in the PDRT maps of $n$ and $G / G_{0}$ that are nearly parallel over about three orders of magnitude in $n$ (Fig. 4).

While the $[\mathrm{O} \mathrm{I}] \lambda 63 \mu \mathrm{m} /[\mathrm{O} \mathrm{I}] \lambda 145 \mu \mathrm{m}$ ratios do not provide further useful constraints on $n$ or $G / G_{0}$ at most locations, near MIR 2 there is a suggestion of a distinct high-density peak in the gas $\left(10^{10.7} \mathrm{~m}^{-3}\right.$, not shown here), albeit with large uncertainty ( $\sim 3$ orders of magnitude in $\log n)$. This is reflected in the slightly betterconstrained three-line localisation shown in Fig. 4, where $\log n=9.5_{-1}^{4.25}$ at MIR 2. To check this, we determined the mass (and thus the density) and luminosity of MIR 2 from SED fitting (§3.3). Assuming MIR 2 is an ellipsoid with a line of sight depth similar to its observable dimensions ( $\sim 9000 \mathrm{au})$, its volumetric mean density is $n=\rho /\left(\mu m_{\mathrm{H}}\right) \approx 8 \times 10^{13} \mathrm{~m}^{-3}(\S 3.3)$, close to PDRT's mean $+1 \sigma$ value of $n$ given the temperature and $G / G_{0}$, and lending credence to the PDRT result despite its large uncertainty.

At these densities, in a relatively weak far-ultraviolet (FUV) field, and given that BYF 73 is on average cool and dense enough to have a CO-depleted center (Pitts et al. 2018, subm.), carbon should be locked up in CO. The measured line luminosities around MIR 2 are $\sim 7 \times 10^{-3} L_{\odot}$ in the $[\mathrm{C}$ II $] \lambda 158 \mu \mathrm{m}$ line and $\sim 4 \times 10^{-3} L_{\odot}$ in the $[\mathrm{O}$ I $] \lambda 145 \mu \mathrm{m}$ line. We conclude that in BYF 73, FIR "cooling" lines of $\mathrm{O}$ and $\mathrm{C}$ do not contribute significantly to the energy balance of the cloud, compared to the total FIR luminosity (§3.3).

\subsection{MIR Point Sources}

The MIR photometry reveals three features. First, we detect only six (proto)stars near the center of the molecular cloud, despite deep, high-resolution Very Large Telescope (VLT) $J H K$ data showing $\sim 30$ heavily reddened objects $(J-K>3)$ in the same $30^{\prime \prime}$-wide area (Andersen et al. 2017). The other two MIR stars, MIR 3 and 8 , are either located within or projected onto the PDR front, and so may be slightly more evolved young stellar objects. The six central MIR stars may be the only true protostars in the imaged area, while the remaining nearinfrared (NIR) objects may just be foreground pre-main sequence stars.

Second, only MIR 1-3 contribute significantly to the bolometric luminosity of the cloud. MIR 3-8 have slowly rising SEDs in the MIR, resembling classic Class II or III protostars (Adams et al. 1987; Barsony 1994; Andre \& Montmerle 1994; MIR 5 seems to be brighter than expected in the IRAC band 4 image, but is the most affected by blending with MIR 2, so this data point should be treated with caution.) Only the SEDs of MIR 1 and 2 show the steep rise at longer wavelengths expected of Class 0 or I protostars, although MIR 1 will need higher-resolution FIR data to separate it cleanly from its much brighter neighbor MIR 2. MIR 2 seems to fall between Class 0 and I definitions: it has a relatively high $T_{\text {bol }}$ and low $L_{\text {submm }} / L_{\text {bol }}$, suggesting Class I, but very high gas fraction and infall rate, plus small age

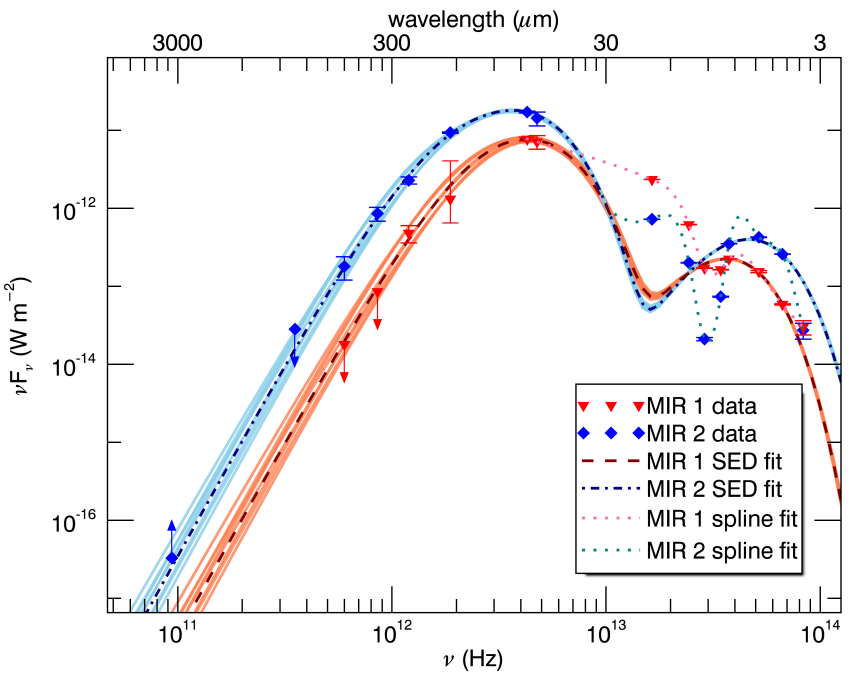

Figure 5. Two-component SED fits to MIR 1 and 2. The model used does not fit the $10 \mu \mathrm{m}$ silicate feature or small grain emission. $t_{\text {inf }}=M / \dot{M} \sim 7000 \mathrm{yr}$, suggesting Class 0 .

Third, all of the MIR stars except MIR 3 and 8 (the two stars possibly within the PDR) show absorption near $10 \mu \mathrm{m}$, attributable to the $9.7 \mu \mathrm{m}$ silicate feature and indicating the presence of intervening or circumstellar cold dust. For MIR 1 and $4-7$, the absorption has a depth to $30-50 \%$ of the adjacent $12 \mu \mathrm{m}$ or $8 \mu \mathrm{m}$ continuum, indicating dust with optical depths at $10 \mu \mathrm{m}$ near 1. For MIR 2, however, the absorption is $>90 \%$ of the MIR continuum, suggesting correspondingly larger amounts of dust, $\tau_{10} \sim 3 \pm 1$. Using a conversion of $A_{V} / \tau_{9.7}=18.5 \pm 1.0$ magnitudes (Mathis 1990), we find approximate values of visual extinction toward each star of $54 \pm 18^{m}$ (MIR 2) or $18 \pm 5^{m}$ (others), though these conversions are for diffuse dust. In molecular clouds, this conversion shows substantial variation - often flattening at large $A_{V}$ (Chiar et al. 2007) - so these extinction estimates are likely lower limits. Assuming further standard conversions of $N_{\mathrm{H}}=1.87 \times 10^{25} \mathrm{~m}^{-2} A_{V} \approx 2 N_{\mathrm{H}_{2}}$ in these clouds (Gerin \& Liszt 2017), where the HI contribution to the total column density is assumed to be small, $\Sigma=1.88 M_{\odot} \mathrm{pc}^{-2}\left(N_{\mathrm{H}_{2}} / 10^{24} m^{-2}\right)$ (Barnes et al. 2018 ), and multiplying by 2 to account for the rear half of each core, we obtain corresponding (very approximate) total mass columns from the silicate absorption of $1950 \pm 650 M_{\odot} \mathrm{pc}^{-2}(\mathrm{MIR} 2)$ and $650 \pm 190 M_{\odot} \mathrm{pc}^{-2}$ (others). If we assume a fiducial envelope size of $10^{4} \mathrm{au}$ (Adams et al. 1987), the total masses (better estimated in $\S 3.3)$ are $\sim 110 \pm 40 M \odot$ for MIR 2 and $\sim 40 \pm 10 M_{\odot}$ for the others. As we show below, this approach is a case in point for the caution urged by Gerin \& Liszt (2017).

\subsection{FIR/Sub-mm Continuum SED Fitting}

To compute the core masses for the MIR point sources, we cropped and regridded the Herschel and SOFIA [O I] $\lambda 63 \mu \mathrm{m}$ continuum images to the area and pixel grid of the FIFI-LS [C II] image, and experimented with fitting up to three 2D Gaussian PSFs at each wavelength, fixed at their respective MIR positions. MIR 1 and 2 are 7 !' 4 apart, fortuitously aligned with the minor axes of the PACS beams, so they are separable at $63 \mu \mathrm{m}$ and $70 \mu \mathrm{m}$, but not at longer wavelengths. MIR 5 is too close to both MIR 1 and 2 to be separable by Herschel or SOFIA, 
but shorter-wavelength data (Fig. 3) already suggest that MIR 3-8 contribute negligibly in the FIR, compared to MIR 1-2; even MIR 3 is undetected as a resolved object for $\lambda>30 \mu \mathrm{m} .{ }^{9}$ At $63 \mu \mathrm{m}$ and $70 \mu \mathrm{m}$, we fit Gaussians and background levels to minimize the residuals at MIR 1 and 2. At longer wavelengths, we estimated the contribution of MIR 1 by comparing the shape of the PSF at MIR 2 on the side facing MIR 1, to that on the opposite side. We then integrated the Gaussian models for each core separately at each wavelength, without the background.

We fit the resulting fluxes with a modified Planck function of the form $I_{\nu}=F_{\nu} / \Omega \approx B_{\nu}\left(T_{\mathrm{d}}\right)\left[1-e^{-\tau}\right]$ with $\tau=\left(\nu / \nu_{0}\right)^{\beta} N_{\mathrm{H}_{2}} \mu m_{\mathrm{H}} \kappa_{0} / \gamma$. Here, $B_{\nu}\left(T_{\mathrm{d}}\right)$ is the Planck function at dust temperature $T_{\mathrm{d}} ; \Omega$ is the solid angle of the source; $\beta$ is the dust emissivity index; $\kappa_{0}$ is the dust opacity coefficient at fiducial frequency $\nu_{0}$ (we use $1200 \mathrm{GHz}) ; N_{\mathrm{H}_{2}}$ is the fitted $\mathrm{H}_{2}$ column density; $\gamma$ is the gas-to-dust mass ratio; $\mu=2.8$ is the mean molecular weight per hydrogen molecule; and $m_{\mathrm{H}}$ is the mass of the hydrogen atom. $T_{\mathrm{d}}$ and $N_{\mathrm{H}_{2}}$ were allowed to vary, while $\gamma$ was fixed at 100 (Beckwith et al. 1990), $\beta$ at 2.0 (Bianchi et al. 1999, and references therein), and $\kappa_{0}=\kappa(350 \mu \mathrm{m}) \approx 0.2 \mathrm{~m}^{2} \mathrm{~kg}^{-1}$ (e.g., Liseau et al. 2015). For MIR 2, we used $\Omega$ from the ATCA interferometry, $14.3 \operatorname{arcsec}^{2}$ (see $\S 2.5$ for dimensions), as a representative core size $\left(D^{2} \Omega=2.09 \times 10^{-3} \mathrm{pc}^{2}\right.$ where $D=2.5 \mathrm{kpc}$ ). MIR 1 is only resolved in the IRAC $3.6 \mu \mathrm{m}$ band, so we estimated MIR 1's deconvolved core dimensions to be $1^{\prime \prime} .9 \times 1^{\prime \prime} 6\left(D^{2} \Omega=5.1 \times 10^{-4} \mathrm{pc}^{2}\right)$, noting that the IRAC $3.6 \mu \mathrm{m}$ filter passband encloses the $3.29 \mu \mathrm{m}$ polycyclic aromatic hydrocarbon $(\mathrm{PAH})$ line, which is correlated with dust emission (Jones et al. 2015).

Two-component SED fitting of ATCA, ASTE, Herschel, SOFIA- $63 \mu \mathrm{m}$, and Spitzer/IRAC data yields $N_{\mathrm{H}_{2}}$ $=6.2_{-1.7}^{+3.6} \times 10^{28} \mathrm{~m}^{-2}$ in $T_{\mathrm{d}}=44.8 \pm 0.4 \mathrm{~K}$ gas for MIR 2 , and $N_{\mathrm{H}_{2}}=2.1_{-0.5}^{+1.3} \times 10^{28} \mathrm{~m}^{-2}$ in $T_{\mathrm{d}}=51 \pm 1 \mathrm{~K}$ gas for MIR 1 (see Fig. 5). We only included additional warm temperature components to verify that they contributed negligibly to the total mass column and to estimate the total luminosity; their parameters are unreliable. The formal errors are based on the standard errors of the model fluxes used in fitting and the reported calibration errors of T-ReCS and the Herschel instruments. The total uncertainty of $N_{\mathrm{H}_{2}}$ may be a factor of two or more due to the uncertainty in $\gamma$ (Reach et al. 2015). The $9.7 \mu \mathrm{m}$ silicate absorption feature is not part of the model, and neither is stochastic heating.

For MIR 2, the above column density corresponds to $\tau_{\mathrm{MIR} 2}(70 \mu \mathrm{m}) \approx 14, A_{V} \sim 7000^{m}$, and $\Sigma_{\mathrm{MIR} 2}=$ $1.2_{-0.4}^{+0.3} \times 10^{5} M_{\odot} \mathrm{pc}^{-2}$, where core the warm component's contribution is negligible. These values look extreme, but over the representative size of MIR 2 at $3 \mathrm{~mm}$, the core mass works out to a reasonable $240_{-50}^{+80} M_{\odot}$. The total luminosity of MIR 2 is $4700_{-500}^{+100} L_{\odot}$, of which about $4500 L_{\odot}$ is from the $T_{\mathrm{d}}=45 \mathrm{~K}$ component alone. Despite being only a few percent of BYF 73's total mass and not much warmer than the clump on average (Pitts et al. 2018), MIR 2 contributes nearly half the total luminosity.

\footnotetext{
9 There is extended FIR/sub-mm structure in the direction of MIR 6-7, but we doubt either of these sources contributes meaningfully to this emission.
}

Deepening the mystery, if $\dot{M}=0.034 \pm 0.017 M_{\odot} \mathrm{yr}^{-1}$ (Barnes et al. 2010) and $R \approx 4500 \mathrm{au}$, the gravitational contraction luminosity is $L_{\mathrm{g}}=G M \dot{M} R^{-1} \approx 260 L_{\odot}$, much more than the line luminosity but still only $\sim 6 \%$ of MIR 2's total.

For MIR 1, by the same analysis, $\tau_{\mathrm{MIR} 1}(70 \mu \mathrm{m}) \sim 5$ and $\Sigma_{\mathrm{MIR} 1} \approx 4_{-2}^{+3} \times 10^{4} \mathrm{M}_{\odot} \mathrm{pc}^{-2}$, corresponding to a core mass of about $20_{-10}^{+20} M_{\odot}$. The cool dust component of MIR 1 contributes $1100 \pm 100 L_{\odot}$ to the total luminosity. The T-ReCS $12 \mu \mathrm{m}$ and $18 \mu \mathrm{m}$ data could only be included in the fit with a spline (which was only used to estimate the total luminosity), but this raised MIR 1's total luminosity to $\sim 1900 L_{\odot}$. These T-ReCS data points cannot trace the same temperature component as the FIR data, because that would indicate a $1 M_{\odot}$ protostar with a $4000 L_{\odot}$ luminosity.

For MIR 3, given the background flux levels in the $\mathrm{Her}$ schel data and its non-detection at $\lambda \geq 30 \mu \mathrm{m}$, MIR 3 can be no more than $\sim 10 \%$ of the mass of MIR 1 . If the same holds for MIR 4-8 as indicated by the FIR nondetections, MIR 2 has nearly 10 times the mass of the other seven objects put together.

\section{CONCLUDING REMARKS}

The mass and volume density of MIR 2 is comparable to the larger of two massive cores in SDC 335 (Peretto et al. 2013), a similar molecular clump to BYF 73 undergoing a massive inflow of gas toward its central objects, but in the case of SDC 335 through a prominent network of accreting filaments. BYF 73 is different because the inflow observed in the single-dish data (resolution $40^{\prime \prime}$ ) is across a cloud structure smooth enough to start resolving out on scales $\lesssim 30^{\prime \prime} \approx \frac{1}{3}$ pc. Furthermore, while the two cores in SDC 335 account for about $10 \%$ of the total mass of that cloud, MIR 2 is potentially $\sim 10 \times$ as massive as MIR 1 and MIR 3-8 combined, yet accounts for only about $1 \%$ of BYF 73's total mass: it is $\gtrsim 98 \%$ gas. This result means BYF 73 may represent an even earlier stage of massive star formation than SDC 335, such that much of the cloud still shows signs of CO freeze-out and has detectable sublimation fronts facing both NGC 3324 and the adjacent compact H II region (Pitts et al. 2018). In the hunt for the elusive transition from starless core to massive Class 0 protostar, the cores in BYF 73 may be the closest yet seen.

We thank the SOFIA crew and Gemini-South \& Spitzer staff for outstanding support of their respective telescopes, Vicki Lowe for help with the ATCA observing, and the anonymous referee for several helpful suggestions which improved the paper. R.L.P. and P.J.B. gratefully acknowledge support from grants NASA-ADAP NNX15AF64G and SOF 04-0061. The Australia Telescope Compact Array is part of the Australia Telescope National Facility which is funded by the Australian Government for operation as a National Facility managed by CSIRO. Based on observations obtained at the Gemini Observatory, which is operated by the Association of Universities for Research in Astronomy, Inc., under a cooperative agreement with the NSF on behalf of the Gemini partnership: the National Science Foundation (United States), the National Research Council (Canada), CONICYT (Chile), Ministerio de Cien- 
cia, Tecnología e Innovación Productiva (Argentina), and Ministério da Ciência, Tecnologia e Inovação (Brazil).

Facilities: ATCA, SOFIA(FIFI-LS), Gemini:South(TReCS), Spitzer(IRAC), Herschel(PACS, SPIRE).

\section{REFERENCES}

Adams, F. C., Lada, C. J., \& Shu, F. H. 1987, ApJ, 312, 788 Andersen, M., Barnes, P. J., Tan, J. C., Kainulainen, J., \& de Marchi, G. 2017, ApJ, 850, 12

Andre, P., \& Montmerle, T. 1994, ApJ, 420, 837

Barnes, P. J., Hernandez, A. K., Muller, E., \& Pitts, R. L. 2018, ApJ, to appear

Barnes, P. J., Hernandez, A. K., O'Dougherty, S. N., Schap, III, W. J., \& Muller, E. 2016, ApJ, 831, 67

Barnes, P. J., Ryder, S. D., O'Dougherty, S. N., et al. 2013, MNRAS, 432, 2231

Barnes, P. J., Yonekura, Y., Ryder, S. D., et al. 2010, MNRAS, 402, 73

Barnes, P. J., Yonekura, Y., Fukui, Y., et al. 2011, ApJS, 196, 12

Barsony, M. 1994, in Astronomical Society of the Pacific

Conference Series, Vol. 65, Clouds, Cores, and Low Mass Stars, ed. D. P. Clemens \& R. Barvainis, 197

Beckwith, S. V. W., Sargent, A. I., Chini, R. S., \& Guesten, R. 1990, AJ, 99, 924

Benjamin, R. A., Churchwell, E., Babler, B. L., et al. 2003 , PASP, 115, 953

Bianchi, S., Davies, J. I., \& Alton, P. B. 1999, A\&A, 344, L1

Chiar, J. E., Ennico, K., Pendleton, Y. J., et al. 2007, ApJ, 666 L73

Cohen, M., Walker, R. G., Carter, B., et al. 1999, AJ, 117, 1864

Colditz, S., Fumi, F., Geis, N., et al. 2012, in Proc. SPIE, Vol. 8446, Ground-based and Airborne Instrumentation for Astronomy IV, 844617

Crutcher, R. M. 2012, ARA\&A, 50, 29

Ezawa, H., Kawabe, R., Kohno, K., \& Yamamoto, S. 2004, in Proc. SPIE, Vol. 5489, Ground-based Telescopes, ed. J. M. Oschmann, Jr., 763

Gerin, M., \& Liszt, H. 2017, A\&A, 600, A48

Gerner, T., Beuther, H., Semenov, D., et al. 2014, A\&A, 563, A97

Griffin, M. J., Abergel, A., Abreu, A., et al. 2010, A\&A, 518, L3
Habing, H. J. 1968, Bull. Astron. Inst. Netherlands, 19, 421

Jones, A. G., Bendo, G. J., Baes, M., et al. 2015, MNRAS, 448, 168

Kaufman, M. J., Wolfire, M. G., \& Hollenbach, D. J. 2006, ApJ, 644,283

Klein, R., Beckmann, S., Bryant, A., et al. 2014, in Proc. SPIE, Vol. 9147, Ground-based and Airborne Instrumentation for Astronomy V, 91472X

Kobayashi, M. I. N., Kobayashi, H., Inutsuka, S.-i., \& Fukui, Y. 2018, PASJ, 70, S59

Liseau, R., Larsson, B., Lunttila, T., et al. 2015, A\&A, 578, A131

Longmore, S. N., Kruijssen, J. M. D., Bastian, N., et al. 2014, Protostars and Planets VI, 291

Mathis, J. S. 1990, ARA\&A, 28, 37

Padoan, P., Pan, L., Haugbølle, T., \& Nordlund, Å. 2016, ApJ, 822, 11

Peretto, N., Fuller, G. A., Duarte-Cabral, A., et al. 2013, A\&A, 555, A 112

Pilbratt, G. L., Riedinger, J. R., Passvogel, T., et al. 2010, A\&A, 518, L1

Pitts, R. L., Barnes, P. J., \& Varosi, F. 2018, MNRAS, subm.

Poglitsch, A., Waelkens, C., Geis, N., et al. 2010, A\&A, 518, L2

Pound, M. W., \& Wolfire, M. G. 2008, in Astronomical Society of the Pacific Conference Series, Vol. 394, Astronomical Data Analysis Software and Systems XVII, ed. R. W. Argyle, P. S. Bunclark, \& J. R. Lewis, 654

Preibisch, T., Roccatagliata, V., Gaczkowski, B., \& Ratzka, T. 2012, A\&A, 541, A132

Reach, W. T., Heiles, C., \& Bernard, J.-P. 2015, ApJ, 811, 118

Rygl, K. L. J., Wyrowski, F., Schuller, F., \& Menten, K. M. 2013, A\&A, 549, A5

Sault, R. J., Teuben, P. J., \& Wright, M. C. H. 1995, in Astronomical Society of the Pacific Conference Series, Vol. 77, Astronomical Data Analysis Software and Systems IV, ed. R. A. Shaw, H. E. Payne, \& J. J. E. Hayes, 433

Telesco, C. M., Pina, R. K., Hanna, K. T., et al. 1998, in Proc. SPIE, Vol. 3354, Infrared Astronomical Instrumentation, ed. A. M. Fowler, 534

Wyrowski, F., Güsten, R., Menten, K. M., et al. 2016, A\&A, 585, A149

Zamora-Avilés, M., \& Vázquez-Semadeni, E. 2014, ApJ, 793, 84 